\title{
eHealth technologies to support nutrition and physical activity behaviors in diabetes self-management
}

This article was published in the following Dove Press journal:

Diabetes, Metabolic Syndrome and Obesity:Targets and Therapy

4 November 2016

Number of times this article has been viewed

\author{
Megan E Rollo' \\ Elroy J Aguiar ${ }^{2}$ \\ Rebecca L Williams' \\ Katie Wynne ${ }^{3}$ \\ Michelle Kriss ${ }^{3}$ \\ Robin Callister ${ }^{4}$ \\ Clare E Collins'
}

'School of Health Sciences, Faculty of Health and Medicine, Priority Research Centre for Physical Activity and Nutrition, University of Newcastle, Callaghan, NSW, Australia; ${ }^{2}$ Department of Kinesiology, School of Public Health and Health Sciences, University of Massachusetts Amherst, Amherst, MA, USA; ${ }^{3}$ Department of Diabetes and Endocrinology, John Hunter Hospital, Hunter New England Health, New Lambton, NSW, Australia; ${ }^{4}$ School of Biomedical Sciences and Pharmacy, Faculty of Health and Medicine, Priority Research Centre for Physical Activity and Nutrition, University of Newcastle, Callaghan, NSW, Australia
Correspondence: Megan E Rollo School of Health Sciences, Faculty of Health and Medicine, Priority Research Centre for Physical Activity and Nutrition, University of Newcastle, ATC Building, Callaghan, NSW 2308, Australia Tel +6I 249215649

Email megan.rollo@newcastle.edu.au

\begin{abstract}
Diabetes is a chronic, complex condition requiring sound knowledge and self-management skills to optimize glycemic control and health outcomes. Dietary intake and physical activity are key diabetes self-management (DSM) behaviors that require tailored education and support. Electronic health (eHealth) technologies have a demonstrated potential for assisting individuals with DSM behaviors. This review provides examples of technologies used to support nutrition and physical activity behaviors in the context of DSM. Technologies covered include those widely used for DSM, such as web-based programs and mobile phone and smartphone applications. In addition, examples of novel tools such as virtual and augmented reality, video games, computer vision for dietary carbohydrate monitoring, and wearable devices are provided. The challenges to, and facilitators for, the use of eHealth technologies in DSM are discussed. Strategies to support the implementation of eHealth technologies within practice and suggestions for future research to enhance nutrition and physical activity behaviors as a part of broader DSM are provided.
\end{abstract}

Keywords: diabetes self-management, eHealth, nutrition, physical activity, smartphones, wearables

\section{Introduction}

The worldwide prevalence of diabetes mellitus has more than doubled over the past three decades and still continues to rise, driven by the growth of an aging population and adverse lifestyle factors that increase body weight and age-specific incidence. ${ }^{1,2}$ Diabetes has been identified as one of the major causes of global mortality, resulting in 1.3 million deaths annually. ${ }^{3}$ In $2014, \sim 422$ million people worldwide had diabetes, ${ }^{2}$ with reports that $85 \%$ of those with diabetes have type 2 diabetes mellitus (T2DM), 10\% type 1 diabetes mellitus (T1DM), and the remaining having gestational diabetes mellitus (GDM). ${ }^{4}$ In the Pacific Islands, a quarter of the population has a diagnosis of diabetes, a prevalence closely followed by that in the Middle East and North Africa. ${ }^{2}$ The People's Republic of China and India have the greatest absolute number of people with diabetes with close to 100 million adults diagnosed with diabetes. ${ }^{5}$ The most rapid rise is also occurring in these two countries accounting for an estimated $36 \%$ of the total global increase. ${ }^{6}$

T1DM is most common in younger populations with an estimated global prevalence of half a million in children and adolescents. ${ }^{7}$ Even in these young patients with T1DM, there is an increasing burden of obesity, ${ }^{8}$ resulting in combined insulin deficiency and insulin resistance or "double diabetes". ${ }^{9}$ Accompanying this is an increase in the preva- 
lence of prediabetes and T2DM in adolescents and young adults, ${ }^{9,10}$ particularly in Asian, Pacific Islander, Indigenous Australian and American, African-American, and Hispanic populations. ${ }^{11-14}$ Young-onset T2DM heralds a higher lifetime risk for diabetes-related complications, ${ }^{15}$ and it is associated with a worse prognosis than T1DM. ${ }^{16}$ The epidemiological shift in presentation has led to an increase in T2DM in women of childbearing age and in pregnancy, and an increase in gestational diabetes. ${ }^{17-19}$ Intrauterine exposure to hyperglycemia results in a higher future risk of obesity and diabetes in offspring ${ }^{20,21}$ and therefore promotes an intergenerational cycle of diabetes fueling the current global epidemic. ${ }^{15}$ This highlights the urgent need for effective interventions.

Diabetes is a complex disease that requires the individual to make daily decisions about food, activity, and medications to achieve their goals. ${ }^{22}$ In T2DM, intensive lifestyle interventions reduce body weight and $\mathrm{HbA} 1 \mathrm{c}$ and improve fitness and cardiovascular risk. ${ }^{23}$ Reduced energy intake and weight loss are associated with substantial reductions in mortality. ${ }^{24,25}$ Regardless of diabetes type, lifestyle decisions are reinforced by provision of the knowledge and skills needed for diabetes self-management (DSM). ${ }^{22,26-28}$

Effective DSM encompasses education that involves the delivery of the knowledge and skills necessary for diabetes self-care and focuses on the four key behaviors of blood glucose monitoring, medication adherence, diet, and physical activity. ${ }^{28}$ In addition to education, effective DSM requires ongoing support to assist the individual to implement and sustain these key behaviors, which help optimize glycemic control. ${ }^{28}$ DSM remains challenging for some patients, ${ }^{29,30}$ and a compromise between health goals and quality of life may arise. ${ }^{31}$ Recent innovations in electronic health (eHealth) technologies may provide the support required to improve DSM, in particular, dietary and physical activity behaviors, and may result in better diabetes outcomes.

The aim of this narrative review is to provide examples of recent eHealth literature and technologies in the context of diet and physical activity for DSM, rather than an exhaustive review of the literature on this topic. First, we summarize the recent use of eHealth in this setting, with a focus on the efficacy of novel technologies that include nutrition and physical activity components for DSM. Technologies that are utilized extensively, as well as emerging technologies that are showing promise for DSM interventions, are the focus of this review. Second, we reflect on the challenges to and facilitators of the use of eHealth technologies for DSM. Finally, we offer practical strategies for implementation as a part of diabetes management and provide suggestions for future research investigating nutrition and physical activity behavioral strategies within DSM.

\section{eHealth technologies to support diet and physical activity in DSM}

Nutrition and physical activity are cornerstones of diabetes management. Decisions relating to food intake are considered as one of the most challenging aspects of diabetes control. ${ }^{32}$ Dietary recommendations for diabetes emphasize an individualized approach to optimizing food and nutrient intakes. ${ }^{32}$ These guidelines focus on eating patterns, portion control, and carbohydrate quality, quantity, and distribution, to optimize glycemic control, in addition to weight status, blood pressure, and plasma lipid profile. ${ }^{32}$ Physical activity guidelines recommend the maintenance of regular aerobic and resistance activities, while minimizing sedentary time. ${ }^{32}$ Both dietary intake and physical activity are strongly influenced by behavior, and therefore self-management skills relative to these behaviors are considered imperative for optimal glycemic control ${ }^{33}$ and consequently lowering the risk of diabetes complications.

The term "eHealth" is broad and refers to the use of information and communication technologies to facilitate and/or deliver health care. ${ }^{34}$ Driven by consumer demand, technologies continue to evolve at a rapid pace, in turn resulting in greater uptake of eHealth. As of 2015, median global Internet and smartphone use was $67 \%$ and $43 \%$, respectively. ${ }^{35}$ While greater use is still more common among developed countries, the rate of adoption within developing countries of both the Internet and smartphones is strong. Since the introduction of the iPhone in 2007, the use of smartphones as a mode to deliver and complement health care has grown rapidly. ${ }^{36}$ Improved accessibility through reduction in costs, coupled with an increase in the capabilities and the ubiquity of these technologies within daily activities, has seen a number of recent evaluations into various eHealth technologies for DSM. ${ }^{37-41}$

Acknowledging the pace at which eHealth technologies change, we focus on the literature from the past 5 years, summarizing established technologies such as web-based programs and mobile phone and smartphone applications used in DSM interventions. We also outline advances in DSM using the novel technologies of computer vision, virtual and augmented reality, video games, and wearable devices.

\section{Web-based programs}

Websites offer a platform for information delivery in various media including written, audio, and video content and may also offer access to social support through forums and chat room facilities. In addition, advances in technology allow 
web-based programs to be accessed via mobile devices such as smartphones and tablet computers. Pereira et $\mathrm{al}^{41}$ examined 14 studies that used primarily Internet-based technologies for the delivery of DSM education including both stand-alone programs and/or those allowing improved access to or contact with health professionals. The review assessed various types of technologies and included aspects of telehealth, but only in the form of asynchronous or delayed interaction with participants. The majority of studies evaluated the efficacy of interventions in terms of changes in glycemic control, whereas changes to dietary intake and physical activity were assessed in less than a quarter of the included studies. Providing diabetes education via the Internet was more effective compared to usual care, with the ability to reach more patients and greater convenience being the features contributing to greater efficacy. However, over time, it was noted that participant engagement waned, and it was suggested that due to the chronic nature of diabetes, longer term interventions should use content and features that can be personalized and adapted to the individual over time. ${ }^{41}$

Recent studies have evaluated the impact of web-based interventions on the knowledge of diet and physical activity in women with $\mathrm{GDM}^{42}$ and related behaviors in T2DM. ${ }^{43}$ In each of these interventions, participants made use of an online program, either to access information or to log diet and physical activity data and receive feedback on their progress. While only $50 \%$ of participants increased their nutrition knowledge, those with less education had a greater improvement compared to those who had completed high school. ${ }^{42}$ Compared to usual care, participants with T2DM who received the computer-assisted intervention, either with or without the support of phone calls, showed greater improvements in their overall score of eating habits, greater reductions in total fat intake, and smaller decreases in physical activity over the 4-month intervention, ${ }^{43}$ with the changes in dietary behavior for the intervention groups maintained at 12 months, whereas physical activity decreased. ${ }^{44}$ Although this type of eHealth technology is a popular choice for DSM, most web-based programs have limited scope in the behavioral strategies used, ${ }^{45}$ and therefore their use may be best to supplement care.

\section{Mobile phones and smartphones}

Portable devices such as mobile (or cell) phones, smartphones, and tablet computers have multiple features that are conducive to DSM, and they are some of the more popular technologies used. ${ }^{46,47}$ Mobile phone text messages can be used for oneway information delivery and/or two-way diabetes counseling support. Smartphones have a number of functions, including Internet connectivity, accelerometers, and global positioning systems, and can host applications that are used for selfmonitoring of dietary and physical activity behaviors.

Text messages delivered via mobile or smartphone continue to be a common strategy for DSM, either alone ${ }^{48,49}$ or in combination with other technologies. ${ }^{50}$ Recent studies conducted in $\mathrm{T}_{2} \mathrm{DM}^{48-50}$ and $\mathrm{T}^{4} \mathrm{DM}^{49}$ evaluated the impact of text messages and demonstrated overall improvements in diet and level of exercise, ${ }^{50}$ increasing weekly exercise and daily consumption of fruits and vegetables, ${ }^{48,49}$ lowering fat consumption, ${ }^{49}$ and enhancing skills in food label reading. ${ }^{48}$ The effects of the text messages on improving the consistency of the desired behaviors were positive, with the number of days participants exercised each week increasing from 2 days to almost 4 days and the percentage of participants who ate fruits and vegetables each day increasing from $61 \%$ to $79 \%{ }^{48}$ Diabetes self-care scores improved for healthy eating and for participation in 30-minute exercise sessions. ${ }^{49}$

A number of recent systematic reviews have summarized the features and content of popular mobile diabetes applications (or apps) available commercially or developed for research. ${ }^{38,39,51,52}$ In general, commercially available apps for DSM are limited in their features, with most consisting of one $(54 \%)$ or two functions (28\%) and a primary purpose of data recording (eg, blood glucose readings). ${ }^{51} \mathrm{~A}$ large proportion $(\sim 60 \%)$ of apps did not include features that cover all four key DSM behaviors (ie, blood glucose monitoring, medication adherence, monitoring dietary intake, and physical activity). ${ }^{52}$ Considerably, more apps contained features relating to diet $(51 \%-75 \%)$ compared to physical activity $(40 \%-50 \%){ }^{38,52}$ In addition, a limited number $(\mathrm{n}=27)$ provided education, while only a very small number $(n=7)$ of these provided personalization of either education or feedback. ${ }^{38}$ Most of the apps in the literature had a positive effect on diabetes outcomes (eg, HbA1c)..$^{52}$ Analysis of popular commercial apps revealed low scores in relation to inclusion of behavioral theories in their design. Apps classified as "diet trackers" scored the highest out of the diet group (25/100) and "exercise trackers" scored the highest out of the exercise group (29/100). The scores were primarily low because the strategies used were not personalized. ${ }^{52}$

With the inclusion of a camera feature, mobile phones and smartphones are increasingly being used for image-based dietary assessment within diabetes management. These image-based records were of similar accuracy to written estimated or weighed food records as a method for quantifying intake among older adults with T2DM and were considered to 
have high usability and acceptability. ${ }^{53,54}$ Computer vision, a form of automated image analysis, has further expanded the capabilities of these types of dietary records for DSM. ${ }^{50,55,56}$ Waki et al ${ }^{56}$ incorporated the smartphone app "FoodLog" into a smartphone platform used for DSM called "DialBetics". Participants were required to take photos of their meal and then the app (via an image recognition algorithm) automatically identified foods contained within the image. The user then selected the most appropriate match from a list of foods in a predeveloped database, which included data on the nutrient profile and portion size of the food item. In addition, DialBetics incorporated a physical activity record (pedometer step, exercise type, and duration), as well as blood glucose, blood pressure, and body weight data collection. ${ }^{56}$ There was moderate compliance with use of the DialBetics platform, and the use of the FoodLog app was more consistent in collecting the morning meal compared to the evening. Users, however, did not always complete the entire meal analysis process $(70 \%$ completed), which compromised their opportunity to receive feedback on their nutrient intake. A positive perception of the effect of the FoodLog app on dietary intake was reported in half of the participants with the remainder expressing dissatisfaction with the length of time needed to complete the process (35 minutes each day). ${ }^{56}$

The "GoCARB" app used plate detection and food recognition algorithms to estimate the carbohydrate content of meals present in images. ${ }^{55}$ When the GoCARB application was tested in 19 adults with T1DM, the estimation error of the carbohydrate content of each meal using manual estimation was $28 \pm 38 \mathrm{~g}$, whereas when using the GoCARB app, the estimation error was reduced to $12 \pm 10 \mathrm{~g}$ of carbohydrate. The system correctly identified $85 \%$ of the food items contained within the meals. ${ }^{57}$ The participants rated the usability highly, but delays in the transmission of data and reliance on an Internet connection were seen as negative elements of the application. ${ }^{57}$

\section{Video games}

Standard exercise or movement-based video games on consumer platforms (eg, Nintendo Wii or Microsoft Xbox) or via purpose-built games for DSM can be used to deliver information and teach self-management behaviors in a fun and engaging way. A number of video games have been developed and evaluated for children and adolescents with T1DM; however, these games also now target adults. The Nintendo Wii Fit Plus, a general fitness video game, was evaluated as a method to improve physical activity in adults with T2DM. ${ }^{58}$ Participants in the intervention group were asked to use the game for 30 minutes per day over a 12-week period. The use of Wii Fit successfully increased physical activity levels in this group. Players were more likely to use the Wii Fit with other players such as family members and partners. ${ }^{58}$ The social media game, "HealthSeeker", was specifically designed for T2DM or individuals at risk of diabetes. Players were set "missions" containing educational components explaining why the behavior was beneficial and what steps can help to achieve dietary and exercise goals. An evaluation of the game showed that players of HealthSeeker completed 3,197 missions and 35,478 actions steps; consumed 17,256 healthy meals and conducted 3,323 exercise activities. This was a significant improvement compared to baseline, with an equal effect in participants with or without diabetes. ${ }^{59}$

\section{Virtual and augmented reality}

Virtual reality is the digital creation of a complete virtual environment that simulates reality (visual and auditory) without sharing all of its physical qualities. In some instances, the user can navigate through and interact with the virtual environment. T2DM self-management education can be delivered in a virtual reality environment or in a traditional in-person format. These methods were compared in a study which used a multidisciplinary team (dietitian, diabetes educator, and nurse practitioner) to deliver care to the participant in an initial individual consult, followed by eight group sessions at weekly intervals. ${ }^{60}$ The eHealth intervention used food exhibits and exercise facilities in a virtual world. The control group were provided with faceto-face sessions conducted in a conference room. Physical activity increased by $18 \%$ in the virtual group, whereas it decreased by $25 \%$ in the face-to-face group; however, there were no differences between the groups in dietary intake.

A study of participants with T2DM by Ruggiero et $\mathrm{al}^{61}$ used a third-party virtual reality platform "Second Life" and a study website for communication, tracking goals, and providing feedback on progress. The virtual reality environment contained visual information (information provided through three-dimensional images), such as tips on label reading, and a learning center delivering written materials and videos including those from the American Diabetes Association. Virtual avatars of health professionals (dietitians and exercise researchers) were used to present ten education sessions on healthy eating and physical activity topics. This included real-time discussions about nutrition held in a supermarket or fast food restaurant. As in the earlier study, the virtual reality intervention resulted in a significant increase in real-life physical activity levels. Changes 
in dietary intake were reported only for participants using the Second Life virtual world, with the only change being a decrease in meat intake. ${ }^{61}$

In contrast to virtual reality, augmented reality is the digital superimposition of virtual elements (usually objects) on the current physical environment to enhance or inhibit the individuals' experience of reality, for example, using portion size guides in smartphone apps. Augmented reality is emerging as a novel method to assist individuals with dietary self-monitoring, in particular, the estimation of the portion size of carbohydrate-containing foods. Augmented reality can be used in a number of ways, for example, users are required to manually retrace food consumed to receive feedback on carbohydrate intake. ${ }^{62}$ The effectiveness of this type of augmented reality feature was tested in patients with T1DM to assess improvements in the accuracy of carbohydrate counting. ${ }^{63}$ Participants also used a smartphone app to log blood glucose levels, insulin, and physical activity. The intervention significantly improved carbohydrate counting accuracy; however, participants identified limitations including the complexity and time taken to draw the foods. This augmented reality system was less effective for whole meals including multiple mixed foods. ${ }^{63}$

\section{Wearable technologies}

Wearable technologies, including pedometers (eg, Yamax Digi-Walker SW200), consumer-grade accelerometers (eg, Fitbit and Jawbone), and smartwatches (eg, Apple Watch, Samsung Gear, and Pebble) are emerging as novel ways to support DSM by self-monitoring physical activity and dietary behaviors and providing the user with immediate feedback, reminders, and alerts. The majority of research has focused on pedometers. In 2014, Qiu et al ${ }^{64}$ conducted a meta-analysis of eleven randomized controlled trials that investigated the relationship between pedometer use, physical activity, and glycemic control in individuals with T2DM. Pedometer use was associated with increased physical activity (an increment of $\sim 1,800 \mathrm{steps} /$ day). Interestingly, studies that included goal setting had a greater impact on increasing physical activity than studies without goal setting. Despite this increase in physical activity, there was no change in $\mathrm{HbAlc}$, even in the subgroup with goal setting included in their intervention. The authors concluded that more research was required to elucidate the benefits of pedometers. In particular, to determine the effect of the volume and intensity of activity on different parameters of glycemic control, such as fasting plasma glucose and $\mathrm{HbAlc}$, in different subsets of patients with diabetes.
Smartwatch technologies have been investigated in recent studies of DSM. Arsand et a ${ }^{65}$ conducted a feasibility trial of the smartwatch "Diabetes Diary" application. The authors developed and trialed a Pebble smartwatch application in a small sample of participants with T1DM. This app allowed an entry to be made directly into the smartwatch, recording carbohydrate intake, physical activity, insulin, and blood glucose level. Diabetes Diary featured a vibrating alert function to remind participants about timing of meals and the need for blood glucose measurement. Participants provided positive feedback for usability and the functionality of the Diabetes Diary in supporting DSM.

\section{Challenges and facilitators to using eHealth technologies for DSM}

A multitude of social, cultural, and economic factors influence DSM, and it is important for the health care provider to identify these so as to best support the patient in reaching their goals. ${ }^{66}$ The use of some technologies may raise concerns in users about lack of personal contact when using remote technology, and it may be perceived as less applicable to those with a disability, or social disadvantage or culturally diverse groups ${ }^{67}$ An individual's eHealth literacy, or their knowledge, comfort, and skills to use various technologies to manage health, is also a vital consideration when contemplating eHealth interventions. ${ }^{68}$ Younger individuals with a higher education level tend to be more eHealth literate ${ }^{69}$ however, elderly patients may successfully adopt new technology with appropriate support. ${ }^{70,71}$ Population levels of eHealth literacy change as new technologies emerge and as social values, norms, and environments evolve.$^{68}$ Therefore, this approach requires the health care provider to be confident and familiar with new technology, ${ }^{67,72}$ have an understanding of the benefits and risks of each type of technology, and recognize that technology may present an additional burden to the patient. ${ }^{73}$ The use of eHealth technologies within clinical practice also presents new challenges and unique requirements in regard to data governance (collection, transmission, sharing, and storage of data) relating to health care. Requirements in relation to the privacy and security of eHealth information are country specific (eg, Health Insurance Portability and Accountability Act within the US), and any technologies used must be compliant with legislation.

Access to technology is improving and allows users to more readily benefit from DSM innovations. Internet usage has exceeded $85 \%$ in the US, UK, Australia, and Canada, but the usage is only $40 \%$ worldwide. ${ }^{74}$ Mobile phone use is projected to reach $70 \%$ of the world's population by 2020 , 
with emerging markets in the Asia Pacific, Middle East, and Africa ${ }^{75}$ paralleling the geographic areas with the most rapidly increasing diabetes prevalence. In addition, as technology has progressed, barriers such as cost have become less significant. For example, consumer-grade wearable activity monitors are now widely available and relatively inexpensive and are considered valid for tracking activity. ${ }^{76}$ Along with blood glucose monitoring technologies (Bluetooth-enabled meters and continuous and flash monitoring), lifestyle technologies can improve motivation, enhance DSM, and allow efficient data sharing with the health care team. Each of these devices provides opportunities to access a greater proportion of the "difficult-to-reach" populations and can build health literacy and social networks, which as a consequence reduce health disparities. ${ }^{61,77-80}$ Importantly, eHealth may offer one of the few options for people with chronic disease in resource-poor environments. ${ }^{81}$ To facilitate effective uptake, it is important to tailor the use of eHealth technologies to the individual. In addition, it is imperative that both the potential advantages and disadvantages of these technologies are discussed with the patient to ensure that the most appropriate technology is selected to support DSM.

\section{Implications for practice and further research}

Best practices for DSM education and training have previously been identified. ${ }^{40}$ Strategies included regular monitoring of behaviors and outcomes, interactivity and multicomponent features, and an acknowledgment of the need to tailor the selection of technology to the patient and their level of experience and lifestyle. ${ }^{40}$ Extending on this concept, we provide suggestions for how the eHealth technologies outlined in this review may be used to address issues of access, personalization, continued support, and patient monitoring in the broader context of DSM education and support. ${ }^{28}$ Although the features and uses of these technologies continue to evolve, it is pertinent to acknowledge their current advantages and disadvantages (Table 1).

Within the studies discussed in this review, most apps did not have the ability to tailor the content to the individual, with a large number displaying features that would not be of any benefit to some individuals with diabetes (eg, insulin adjustment for T2DM controlled by diet only). ${ }^{38}$ If app selection is tailored to the individual needs, motivations, and preferences, it may support more meaningful use, particularly if there are tools available to guide clinicians in this process. ${ }^{39}$ Given the chronic nature of diabetes and the need for daily support, priority should be given to the evaluation of features that adapt content and feedback in response to user progress to maximize patient engagement over the long term. This could be achieved through adaptive interventions that assign different components of a program to different individuals based on identified characteristics, and which then alter the frequency of contact in response to the individual's progress. ${ }^{82}$ However, the majority of eHealth technologies in this review simply documented data, with minimal or no ability to use these data to guide patients in making appropriate behavior changes, ${ }^{51}$ therefore, this is an area of DSM research that requires attention.

The synthesis of multiple data on patterns of diet and physical activity combined with real-time physiological tracking (eg, via continuous glucose monitoring devices), and contextual (eg, location) information, provides a "digital data ecosystem" with potential to support DSM, in particular T1DM. ${ }^{83}$ Recent focus has shifted toward designing strategies that use the sensors (eg, global positioning systems) within smartphones and other wearable devices (eg, smartwatch) to allow for "just-in-time" interventions delivered when the individual needs it most. ${ }^{84}$ Through sensor technologies, and the linking of sequenced automated decisions and immediate feedback, just-in-time adaptive interventions may further enhance the level of personalization in relation to eating and activity behaviors. These types of interventions show promise for adoption within DSM education and support strategies.

The use of eHealth technologies by patients for DSM must be practical and value-add to care for both the patient and their clinician. While the number of diabetes-specific smartphone apps has increased rapidly in recent years, limitations exist around their role in diabetes management. Currently, these technologies are still best placed to complement traditional patient-clinician contact, rather than to be used exclusively as a treatment option. Opportunities may arise in the future to combine different technologies (eg, web-based program with smartphone app) with synchronous telehealth, such as video or telephone calls, to mitigate access barriers to one-on-one DSM education and support. However, further improvements in personalization, decision support components, and communication between multiple technologies/ software are needed to support their standalone use. ${ }^{37-41,51,52}$

To date, most of the evidence relating to eHealth for DSM supports more established technologies such as text messaging, web-based programs, and smartphone apps. The use of more interactive and sophisticated technologies, such as computer vision, virtual and augmented reality, and video 
Table I Advantages and disadvantages of eHealth technologies

\begin{tabular}{|c|c|c|}
\hline Technology & Advantages & Disadvantages \\
\hline \multicolumn{3}{|c|}{ Mobile phones and smartphones } \\
\hline Text messages & $\begin{array}{l}\text { Broad reach due to ubiquitous use of cell/mobile phones } \\
\text { and smartphones and ability to be sent across multiple } \\
\text { operating systems } \\
\text { Messages can be personalized to the individual }\end{array}$ & $\begin{array}{l}\text { Character count ( } \mathrm{n}=160 \text { ) per message limits detailed } \\
\text { messages (although multiple messages can be sent) } \\
\text { Although other content can be sent (eg, audio or video), } \\
\text { individuals may have restrictions on the type of content that } \\
\text { can be received (ie, text only) }\end{array}$ \\
\hline & $\begin{array}{l}\text { Useful as an adjunct (booster) with other technology types } \\
\text { Relatively less expensive to set up and deliver compared } \\
\text { to other technologies }\end{array}$ & $\begin{array}{l}\text { Interaction may not be offered (ie, one way) or may be } \\
\text { limited (ie, single word replies) due to limited automation }\end{array}$ \\
\hline Applications (apps) & $\begin{array}{l}\text { Portability of smartphones and associated mobile devices } \\
\text { allows immediate access to apps } \\
\text { Ability to push notifications and reminders based on data } \\
\text { entered or collected passively } \\
\text { Ability to link data with other apps and/or devices } \\
\text { (eg, through the use of API) } \\
\text { Offline functionality is common, however, it is dependent } \\
\text { on the features of the app }\end{array}$ & $\begin{array}{l}\text { If using a third-party app, no control over the timing or type } \\
\text { of content and/or feature changes } \\
\text { If developing an app, ongoing costs associated with } \\
\text { maintenance and updates to software (eg, update of operating } \\
\text { system) } \\
\text { Some apps are only available on selected operating systems } \\
\text { (eg, Apple's iOS) which may limit accessibility }\end{array}$ \\
\hline Web-based programs & $\begin{array}{l}\text { Flexibility in the content and features including capability } \\
\text { for multimedia content and peer and health professional } \\
\text { support } \\
\text { Can be used across multiple devices with Internet access } \\
\text { (eg, computer through to smartphone); however, user } \\
\text { experience may differ across the different devices }\end{array}$ & $\begin{array}{l}\text { Requires Internet connection with most having limited or no } \\
\text { offline capacity }\end{array}$ \\
\hline Video games & $\begin{array}{l}\text { Most commonly used for promoting exercise } \\
\text { Increased accessibility in the home setting removes } \\
\text { some barriers to participation, eg, transport, cost of gym } \\
\text { membership, and stigma associated with exercising at } \\
\text { a gym }\end{array}$ & $\begin{array}{l}\text { Requires specific technology (eg, video game console) } \\
\text { Largely generic and unsupervised; may not account for } \\
\text { variations in an individual's ability to exercise }\end{array}$ \\
\hline Virtual reality & $\begin{array}{l}\text { Simulation of real-world scenarios can be used to } \\
\text { deliver information and train patients in self-management } \\
\text { behaviors providing an immersive, yet controlled } \\
\text { environment to rehearse behaviors }\end{array}$ & $\begin{array}{l}\text { Requires specific technology (eg, headset) } \\
\text { Generic simulation of real-world scenarios may not exactly } \\
\text { translate to individuals' lived experience with diabetes }\end{array}$ \\
\hline Augmented reality & $\begin{array}{l}\text { Provides information to support decision making in the } \\
\text { context of real-life situations } \\
\text { Able to be used on common devices (eg, smartphones) }\end{array}$ & $\begin{array}{l}\text { Virtual content tends to be built for specific situations; } \\
\text { therefore, the use may be limited to selected environments }\end{array}$ \\
\hline $\begin{array}{l}\text { Wearable } \\
\text { technologies }\end{array}$ & $\begin{array}{l}\text { Conveniently located and immediate feedback on behaviors } \\
\text { (eg, step/day) } \\
\text { Ability to program alerts and reminders (eg, sedentary } \\
\text { time) }\end{array}$ & $\begin{array}{l}\text { Battery life can vary between devices } \\
\text { Limited information available on device itself, requires pairing } \\
\text { with computer/smartphone }\end{array}$ \\
\hline
\end{tabular}

Abbreviations: API, application programming interface; eHealth, electronic health.

games, has in the past been restricted by the need for nonportable, expensive equipment. However, uptake is likely to surge with virtual reality headsets now becoming more widely available for consumers (eg, Oculus Rift, Samsung Gear VR, and Sony PlayStation VR). ${ }^{85}$ As a result, opportunities will arise for clinicians, researchers, and industry to be involved in the development, implementation, and evaluation of virtual reality environments for the support of DSM behaviors.

The effect of combined asynchronous (static or delayed interaction, eg, website and email) and synchronous (real time, eg, telephone and video calls) technologies on the efficacy of DSM warrants further investigation. ${ }^{37,41}$ With regard to self-monitoring of diet and physical activity to inform DSM, questions remain as to the optimal dosage (amount, frequency, or intensity) necessary to enhance glycemic control. Determining the optimal sequence of eHealth-based strategies for DSM at the individual level can be achieved through study designs such as a sequential multiple assignment randomized trial (SMART). Compared to randomized controlled trials that aim to evaluate the effect of an existing intervention, SMART designs aim to optimize the intervention arm. ${ }^{86}$ The use of these study designs to 
optimize eHealth components and determine the ideal dosage as part of an adaptive intervention is gaining momentum in obesity management settings $;{ }^{87}$ however, studies of this type for DSM are yet to be undertaken.

\section{Conclusion}

This review summarizes recent evidence regarding a number of technologies to support DSM in relation to diet and physical activity. Text messages, smartphone apps, and web-based programs can have positive impacts on DSM behaviors. Although still in their infancy, computer vision, video games, wearables, and augmented and virtual reality demonstrate potential as another opportunity to support DSM through automation and immersion; however, individuals should be adequately trained in the use of these technologies. Practice recommendations include tailoring the use of eHealth technologies to an individual's DSM needs and preferences, while further data on the efficacy, usability, and acceptability of the more novel technologies is required. Research into the optimization of these technologies (including types and dosage) to support diet and physical activity behaviors in the context of adaptive DSM interventions is warranted.

\section{Disclosure}

The authors report no conflicts of interest in this work.

\section{References}

1. Chen L, Magliano DJ, Zimmet PZ. The worldwide epidemiology of type 2 diabetes mellitus - present and future perspectives. Nat Rev Endocrinol. 2012;8(4):228-236.

2. NCD Risk Factor Collaboration. Worldwide trends in diabetes since 1980: a pooled analysis of 751 population-based studies with 4.4 million participants. Lancet. 2016;387(10027):1513-1530.

3. Naghavi M, Wang H, Lozano R, et al. Global, regional, and national age-sex specific all-cause and cause-specific mortality for 240 causes of death, 1990-2013: a systematic analysis for the Global Burden of Disease Study 2013. Lancet. 2015;385(9963):117-171.

4. Diabetes Australia. Diabetes in Australia; 2015. Available from https:// www.diabetesaustralia.com.au/diabetes-in-australia. Accessed May 18, 2016.

5. Xu Y, Wang L, He J, et al; 2010 China Noncommunicable Disease Surveillance Group. Prevalence and control of diabetes in Chinese adults. JAMA. 2013;310(9):948-959.

6. Shaw JE, Sicree RA, Zimmet PZ. Global estimates of the prevalence of diabetes for 2010 and 2030. Diabetes Res Clin Pract. 2010;87(1): 4-14.

7. Hannon TS, Arslanian SA. The changing face of diabetes in youth: lessons learned from studies of type 2 diabetes. Ann N Y Acad Sci. 2015;1353(1):113-137.

8. Kaminski BM, Klingensmith GJ, Beck RW, et al. Body mass index at the time of diagnosis of autoimmune type 1 diabetes in children.JPediatr. 2013; 162(4):736-740.

9. Cleland S, Fisher B, Colhoun H, Sattar N, Petrie J. Insulin resistance in type 1 diabetes: what is 'double diabetes' and what are the risks? Diabetologia. 2013;56(7):1462-1470.
10. Dabelea D, Mayer-Davis EJ, Saydah S, et al; SEARCH for Diabetes in Youth Study. Prevalence of type 1 and type 2 diabetes among children and adolescents from 2001 to 2009. JAMA. 2014;311(17): $1778-1786$.

11. Kitagawa T, Owada M, Urakami T, Yamauchi K. Increased incidence of non-insulin dependent diabetes mellitus among Japanese schoolchildren correlates with an increased intake of animal protein and fat. Clin Pediatr. 1998;37(2):111-115.

12. SEARCH for Diabetes in Youth Study Group; Liese AD, D'Agostino RB Jr, Hamman RF, et al. The burden of diabetes mellitus among US youth: prevalence estimates from the SEARCH for diabetes in youth study. Pediatrics. 2006;118(4):1510-1518.

13. Dabelea D, Bell RA, D'Agostino RB Jr, et al. Incidence of diabetes in youth in the United States. JAMA. 2007;297(24):2716-2724.

14. Li C, Ford ES, Zhao G, Mokdad AH. Prevalence of pre-diabetes and its association with clustering of cardiometabolic risk factors and hyperinsulinemia among US adolescents National Health and Nutrition Examination Survey 2005-2006. Diabetes Care. 2009;32(2): 342-347.

15. Hillier TA, Pedula KL, Schmidt MM, Mullen JA, Charles M-A, Pettitt DJ. Childhood obesity and metabolic imprinting the ongoing effects of maternal hyperglycemia. Diabetes Care. 2007;30(9):2287-2292.

16. Constantino MI, Molyneaux L, Limacher-Gisler F, et al. Long-term complications and mortality in young-onset diabetes type 2 diabetes is more hazardous and lethal than type 1 diabetes. Diabetes Care. 2013;36(12):3863-3869.

17. Dabelea D, Snell-Bergeon JK, Hartsfield CL, et al; Kaiser Permanente of Colorado GDM Screening Program. Increasing prevalence of gestational diabetes mellitus (GDM) over time and by birth Cohort Kaiser Permanente of Colorado GDM screening program. Diabetes Care. 2005;28(3):579-584.

18. Lawrence JM, Contreras R, Chen W, Sacks DA. Trends in the prevalence of preexisting diabetes and gestational diabetes mellitus among a racially/ethnically diverse population of pregnant women, 1999-2005. Diabetes Care. 2008;31(5):899-904.

19. Getahun D, Nath C, Ananth CV, Chavez MR, Smulian JC. Gestational diabetes in the United States: temporal trends 1989 through 2004. Am J Obstet Gynecol. 2008;198(5):e521-e525.

20. Pettitt DJ, Baird HR, Aleck KA, Bennett PH, Knowler WC. Excessive obesity in offspring of Pima Indian women with diabetes during pregnancy. $N$ Engl J Med. 1983;308(5):242-245.

21. Dabelea D, Hanson RL, Lindsay RS, et al. Intrauterine exposure to diabetes conveys risks for type 2 diabetes and obesity: a study of discordant sibships. Diabetes. 2000;49(12):2208-2211.

22. Powers MA, Bardsley J, Cypress M, et al. Diabetes self-management education and support in type 2 diabetes a joint position statement of the American Diabetes Association, the American Association of diabetes educators, and the academy of nutrition and dietetics. Diabetes Educ. 2015;41(4):417-430.

23. Look AHEAD Research Group; Wing RR, Bolin P, Brancati FL, et al. Cardiovascular effects of intensive lifestyle intervention in type 2 diabetes. N Engl J Med. 2013;369(2):145.

24. Williamson DF, Thompson TJ, Thun M, Flanders D, Pamuk E, Byers T. Intentional weight loss and mortality among overweight individuals with diabetes. Diabetes Care. 2000;23(10):1499-1504.

25. Gregg EW, Gerzoff RB, Thompson TJ, Williamson DF. Trying to lose weight, losing weight, and 9-year mortality in overweight US adults with diabetes. Diabetes Care. 2004;27(3):657-662.

26. Waugh N, Cummins E, Royle P, et al. Newer agents for blood glucose control in type 2 diabetes: systematic review and economic evaluation. Health Technol Assess. 2010;14(36):1-248.

27. Association AD. Standards of medical care in diabetes - 2010. Diabetes Care. 2010;33(suppl 1):S11-S61.

28. Haas L, Maryniuk M, Beck J, et al. National standards for diabetes selfmanagement education and support. Diabetes Care. 2013;36(suppl 1): S100-S108. 
29. Savoca MR, Miller CK, Quandt SA. Profiles of people with type 2 diabetes mellitus: the extremes of glycemic control. Soc Sci Med. 2004;58(12):2655-2666.

30. Forbes A. The Organisation \& Delivery of Diabetes Services in the UK: A Scoping Exercise. London: School of Nursing and Midwifery, King's College London; 2010.

31. Frost J, Garside R, Cooper C, Britten N. A qualitative synthesis of diabetes self-management strategies for long term medical outcomes and quality of life in the UK. BMC Health Serv Res. 2014;14(1):1.

32. American Diabetes Association. 3. Foundations of care and comprehensive medical evaluation. Diabetes Care. 2016;39(suppl 1):S23-S35.

33. Haas L, Maryniuk M, Beck J, et al. National standards for diabetes self-management education and support. Diabetes Care. 2012;35(11):2393-2401.

34. van Gemert-Pijnen EWCJ, Nijland N, van Limburg M, et al. A holistic framework to improve the uptake and impact of eHealth technologies. J Med Internet Res. 2011;13(4):e111.

35. Poushter $J$ [webpage on the Internet]. Smartphone Ownership and Internet Usage Continues to Climb in Emerging Economies; 2016. Available from: http://www.pewglobal.org/2016/02/22/smartphone-ownershipand-internet-usage-continues-to-climb-in-emerging-economies/. Accessed May 16, 2016

36. Fiordelli M, Diviani N, Schulz PJ. Mapping mHealth research: a decade of evolution. J Med Internet Res. 2013;15(5):e95.

37. El-Gayar O, Timsina P, Nawar N, Eid W. A systematic review of IT for diabetes self-management: are we there yet? Int J Med Inform. 2013;82(8):637-652.

38. Chomutare T, Fernandez-Luque L, Arsand E, Hartvigsen G. Features of mobile diabetes applications: review of the literature and analysis of current applications compared against evidence-based guidelines. $J$ Med Internet Res. 2011;13(3):e65.

39. Hale K, Capra S, Bauer J. A framework to assist health professionals in recommending high-quality apps for supporting chronic disease self-management: illustrative assessment of type 2 diabetes apps. JMIR Mhealth Uhealth. 2015;3(3):e87.

40. Fitzner K, Moss G. Telehealth - an effective delivery method for diabetes self-management education? Popul Health Manag. 2013;16(3):169-177.

41. Pereira K, Phillips B, Johnson C, Vorderstrasse A. Internet delivered diabetes self-management education: a review. Diabetes Technol Ther. 2015;17(1):55-63.

42. Carolan-Olah M, Steele C, Krenzin G. Development and initial testing of a GDM information website for multi-ethnic women with GDM. BMC Pregnancy Childbirth. 2015;15:145.

43. Glasgow RE, Kurz D, King D, et al. Outcomes of minimal and moderate support versions of an internet-based diabetes self-management support program. J Gen Intern Med. 2010;25(12):1315-1322.

44. Glasgow RE, Kurz D, King D, et al. Twelve-month outcomes of an Internet-based diabetes self-management support program. Patient Educ Couns. 2012;87(1):81-92.

45. van Vugt M, de Wit M, Cleijne WHJJ, Snoek FJ. Use of behavioral change techniques in web-based self-management programs for type 2 diabetes patients: systematic review. J Med Internet Res. 2013;15(12):e279.

46. Smith A [webpage on the Internet]. U.S. Smartphone Use in 2015; 2016. Available from: http://www.pewinternet.org/2015/04/01/ussmartphone-use-in-2015/. Accessed May 16, 2016.

47. Perez $\mathrm{S}$ [webpage on the Internet]. Consumers spend $85 \%$ of time on smartphones in apps, but only 5 apps see heavy use; 2015. Available from: http://techcrunch.com/2015/06/22/consumers-spend-85-of-timeon-smartphones-in-apps-but-only-5-apps-see-heavy-use/. Accessed May 16, 2016.

48. Burner E, Menchine M, Taylor E, Arora S. Gender differences in diabetes self-management: a mixed-methods analysis of a mobile health intervention for inner-city Latino patients. J Diabetes Sci Technol. 2013;7(1):111-118.

49. Nundy S, Mishra A, Hogan P, Lee SM, Solomon MC, Peek ME. How do mobile phone diabetes programs drive behavior change? Evidence from a mixed methods observational cohort study. Diabetes Educ. 2014;40(6):806-819
50. Waki K, Fujita H, Uchimura Y, et al. DialBetics: a novel smartphonebased self-management support system for type 2 diabetes patients. $J$ Diabetes Sci Technol. 2014;8(2):209-215.

51. Arnhold M, Quade M, Kirch W. Mobile applications for diabetics: a systematic review and expert-based usability evaluation considering the special requirements of diabetes patients age 50 years or older. $J$ Med Internet Res. 2014;16(4):e104.

52. El-Gayar O, Timsina P, Nawar N, Eid W. Mobile applications for diabetes self-management: status and potential. J Diabetes Sci Technol. 2013;7(1):247-262.

53. Rollo ME, Ash S, Lyons-Wall P, Russell A. Trial of a mobile phone method for recording dietary intake in adults with type 2 diabetes: evaluation and implications for future applications. JTelemed Telecare. 2011;17(6):318-323.

54. Rollo ME, Ash S, Lyons-Wall P, Russell AW. Evaluation of a mobile phone image-based dietary assessment method in adults with type 2 diabetes. Nutrients. 2015;7(6):4897-4910.

55. Anthimopoulos M, Dehais J, Shevchik S, et al. Computer vision-based carbohydrate estimation for type 1 patients with diabetes using smartphones. J Diabetes Sci Technol. 2015;9(3):507-515.

56. Waki K, Aizawa K, Fujita H, et al. DialBetics with a multimedia food recording tool, FoodLog: smartphone based self-management for type 2 diabetes. Diabetes Technol Therap. 2015;17:A136-A137.

57. Rhyner D, Loher H, Dehais J, et al. Carbohydrate estimation by a mobile phone-based system versus self-estimations of individuals with type 1 diabetes mellitus: a comparative study. J Med Internet Res. 2016;18(5):e101.

58. Kempf K, Martin S. Autonomous exercise game use improves metabolic control and quality of life in type 2 diabetes patients - a randomized controlled trial. BMC Endocr Disord. 2013;13(1):57-75.

59. Campbell A, Fernandez M, Mensign C, Imershein S. Health Games: Engaging People in Positive Lifestyle and Nutrition Behaviours through Social Media; Practical Diabetology. 2012;31(1):30-32.

60. Rosal MC, Heyden R, Mejilla R, Rizzo DePaoli M, Veerappa C, Wiecha JM. Design and methods for a comparative effectiveness pilot study: virtual world vs. face-to-face diabetes self-management. JMIR Res Protoc. 2012;1(2):e24.

61. Ruggiero L, Moadsiri A, Quinn LT, et al. Diabetes island: preliminary impact of a virtual world self-care educational intervention for African Americans with type 2 diabetes. JMIR Serious Games. 2014;2(2):e10.

62. Stutz T, Dinic R, Domhardt M, Ginzinger S. Can mobile augmented reality systems assist in portion estimation? A user study. Paper presented at: 2014 IEEE International Symposium on Mixed and Augmented Reality - Media, Art, Social Science, Munich, Germany. Humanities and Design (ISMAR-MASH'D); September 10-12, 2014.

63. Domhardt M, Tiefengrabner M, Dinic R, et al. Training of carbohydrate estimation for people with diabetes using mobile augmented reality. $J$ Diabetes Sci Technol. 2015;9(3):516-524.

64. Qiu S, Cai X, Chen X, Yang B, Sun Z. Step counter use in type 2 diabetes: a meta-analysis of randomized controlled trials. BMC Med. 2014;12:36.

65. Arsand E, Muzny M, Bradway M, Muzik J, Hartvigsen G. Performance of the first combined smartwatch and smartphone diabetes diary application study. J Diabetes Sci Technol. 2015;9(3):556-563.

66. Wilkinson A, Whitehead L, Ritchie L. Factors influencing the ability to self-manage diabetes for adults living with type 1 or 2 diabetes. Int J Nurs Stud. 2014;51(1):111-122.

67. Dobson KG, Hall P. A pilot study examining patient attitudes and intentions to adopt assistive technologies into type 2 diabetes selfmanagement. J Diabetes Sci Technol. 2015;9(2):309-315.

68. Norman DC, Skinner AH. eHealth literacy: essential skills for consumer health in a networked world. $J$ Med Internet Res. 2006;8(2):e9.

69. Neter E, Brainin E. eHealth literacy: extending the digital divide to the realm of health information. J Med Internet Res. 2012;14(1):e19.

70. Luijkx K, Peek S, Wouters E. "Grandma, you should do it - it's cool" older adults and the role of family members in their acceptance of technology. Int J Environ Res Public Health. 2015;12(12):15470-15485.

71. Tan CCL, Cheng KKF, Wang W. Self-care management programme for older adults with diabetes: an integrative literature review. Int $J$ Nurs Pract. 2015;21(S2):115-124. 
72. Vuong AM, Huber JC Jr, Bolin JN, et al. Factors affecting acceptability and usability of technological approaches to diabetes self-management: a case study. Diabetes Technol Ther. 2012;14(12):1178-1182.

73. Ancker JS, Witteman HO, Hafeez B, Provencher T, Van de Graaf M, Wei E. "You get reminded you're a sick person": personal data tracking and patients with multiple chronic conditions. J Med Internet Res. 2015;17(8):e202.

74. The World Bank [webpage on the Internet]. Internet users as percentage of population; 2016. Available from: https:/www.google.com/ publicdata/explore?ds=d5bncppjof8f9_\&met_y=it_net_user_p2\&hl= en $\& \mathrm{dl}=$ en \&idim $=$ country:USA:CAN:GBR\#! ctype $=1 \&$ strail=false $\& b$ $\mathrm{cs}=\mathrm{d} \& n$ selm $=\mathrm{h} \& \mathrm{met} \_\mathrm{y}=\mathrm{it} \_$net_user_p2\&scale_y=lin\&ind_y=false \& rdim=region\&idim=country:USA:CAN:GBR:AUS\&ifdim=region\&t $\operatorname{dim}=$ true\&hl=en_US\&dl=en\&ind=false. Accessed May 3, 2016.

75. Lunden I [webpage on the Internet]. 6.1B Smartphone Users Globally By 2020, Overtaking Basic Fixed Phone Subscriptions; 2015. Available from: http://techcrunch.com/2015/06/02/6-1b-smartphoneusers-globally-by-2020-overtaking-basic-fixed-phone-subscriptions/. Accessed May 4, 2016.

76. Evenson KR, Goto MM, Furberg RD. Systematic review of the validity and reliability of consumer-wearable activity trackers. Int J Behav Nutr Phys Act. 2015;12:159.

77. Gibbons MC, Fleisher L, Slamon RE, Bass S, Kandadai V, Beck JR. Exploring the potential of web 2.0 to address health disparities. J Health Commun. 2011;16(sup1):77-89.

78. Roblin DW. The potential of cellular technology to mediate social networks for support of chronic disease self-management. $J$ Health Commun. 2011;16(sup1):59-76.
79. Rosal MC, Heyden R, Mejilla R, et al. A virtual world versus face-toface intervention format to promote diabetes self-management among African American women: a pilot randomized clinical trial. JMIR Res Protoc. 2014;3(4):e54.

80. Wayne N, Perez DF, Kaplan DM, Ritvo P. Health coaching reduces HbA1c in type 2 diabetic patients from a lower-socioeconomic status community: a randomized controlled trial. $J$ Med Internet Res. 2015;17(10):e224.

81. Lucas H. New technology and illness self-management: potential relevance for resource-poor populations in Asia. Soc Sci Med. 2015;145:145-153.

82. Collins LM, Murphy SA, Bierman KL. A conceptual framework for adaptive preventive interventions. Prev Sci. 2004;5(3):185-196.

83. Heintzman ND. A digital ecosystem of diabetes data and technology: services, systems, and tools enabled by wearables, sensors, and apps. J Diabetes Sci Technol. 2016;10(1):35-41.

84. Spruijt-Metz D, Wen CK, O'Reilly G, et al. Innovations in the use of interactive technology to support weight management. Curr Obes Rep. 2015;4(4):510-519.

85. Collins K. Eye tracking in VR headsets is the future, and it's starting now. 2016. Accessed May, 2016.

86. Almirall D, Nahum-Shani I, Sherwood NE, Murphy SA. Introduction to SMART designs for the development of adaptive interventions: with application to weight loss research. Transl Behav Med. 2014;4(3):260-274.

87. Sherwood NE, Butryn ML, Forman EM, et al. The BestFIT trial: a SMART approach to developing individualized weight loss treatments. Contemp Clin Trials. 2016;47:209-216.

\section{Publish your work in this journal}

Diabetes, Metabolic Syndrome and Obesity: Targets and Therapy is an international, peer-reviewed open-access journal committed to the rapid publication of the latest laboratory and clinical findings in the fields of diabetes, metabolic syndrome and obesity research. Original research, review, case reports, hypothesis formation, expert opinion and commentaries are all considered for publication. The manuscript management system is completely online and includes a very quick and fair peer-review system, which is all easy to use. Visit http://www.dovepress.com/testimonials.php to read real quotes from published authors. 